\title{
Hustende und schnupfende Kinder
}

\author{
Wie kommen wir über den Winter?
}

\begin{abstract}
Corona hat unser Leben verändert. Der Umgang damit verlangt uns einiges ab. Und Corona wird uns weiterhin begleiten, somit sind wir gefordert, ein Leben mit und trotz Corona zu gestalten, bis vielleicht wieder ein "Leben nach Corona" möglich ist. Damit befasste sich eine Pressekonferenz der ÖGKJ, die im September in Wien stattfand.

„Als Kinder- und Jugendärzt*innen sehen wir uns verpflichtet, unser Wissen um Infektionen und Krankheiten im Allgemeinen den Kindergärten, Schulen und anderen Institutionen verfügbar zu machen", so Ao. Prof. Dr. Daniela Karall, Präsidentin der ÖGKJ, und weiter: „Erfreulicherweise wurde dies seitens der zuständigen Ministerien und Gesundheitsbehörden in den letzten Monaten auch wiederholt in Anspruch genommen."
\end{abstract}

\section{Wer, wenn nicht wir?}

Kinder- und Jugendärztinnen und -ärzte haben Maßnahmen zum Umgang mit Infektionskrankheiten gelernt und bereits lange vor Corona praktiziert. Daher gehören sie zu jenen, die vormachen und zeigen können/ müssen, wie gedeihliches und sicheres Miteinander gelingen kann, besonders wenn es um die Altersgruppe der 0 - bis 18-Jährigen geht.

Die notwendige Disziplin in der Umsetzung von Hygienemaßnahmen und Kontaktreduktion ist dabei der Meinung der ÖGKJ nach der Schlüssel. Sie ist auch bei Kindern und Jugendlichen ausreichend vorhanden, v.a. wenn auch die Eltern notwendige Maßnahmen verständnisvoll mittragen.

Essentiell ist bei allem Umgang mit (nicht nur) Corona, dass sorgfältig und besonnen agiert wird, aber nicht Furcht und Angst das Geschehen bestimmen.

\section{Persönlicher Kontakt und soziale Integration}

Ein geregelter Tagesablauf und der persönliche Kontakt zu Gleichaltrigen und Lehrpersonen sind essentiell für die Entwicklung von Kindern und Jugendlichen. Sprache und Kontakt machen menschliche Beziehungen aus. Mimik, Gestik, ein Blick in die Augen verraten uns, wie sich jemand fühlt. Diese wichtigen Elemente der sozialen Entwicklung und Integration können durch virtuelle Medien nicht ersetzt werden und müssen daher - wann immer möglich - den Kindern und Jugendlichen erhalten bleiben. Aus diesem Grund ist es, so Karall, der ÖGKJ ein Anliegen, mit ihrer Expertise in die Entscheidungsprozesse um Kindergarten und Schule weiterhin einbezogen zu werden.

\section{COVID-19 bei Kindern und Jugendlichen}

Schon früh in der SARS-CoV-2 Pandemie hat sich gezeigt, dass Kinder bei dieser Infektionserkrankung eine besondere Stellung einnehmen, indem sie seltener und in der Regel wesentlich milder erkranken oder gar keine Symptome zeigen. Diese Tatsache-wie auch das Wissen von anderen Atemwegs-Infektionen - hat zu der falschen Annahme geführt, dass Kinder in der Übertragung eine überdurchschnittlich große Rolle spielen. „Dies wurde jedoch nie bewiesen, vielmehr deuten immer mehr Studien darauf hin, dass Kinder nicht nur seltenerer Symptome entwickeln, sondern sich auch seltener infizieren, z. B. bei erkrankten Personen im selben Haushalt", berichtete PD Dr. Volker Strenger, AG-Leiter "Infektiologie" der ÖGKJ.

Studien haben - teilweise sehr medienwirksam - berichtet, dass Kinder gleich viele Viren ausscheiden würden wie Erwachsene. Diese Studien haben jedoch nur die Menge an Virus-Erbsubstanz auf den Abstrichtupfern im Labor untersucht und nicht die Menge an ausgeschiedenen Viren. Viel mehr haben Cluster-Analysen - u.a. auch in Schulen - gezeigt, dass Kinder seltener Indexfälle sind und dass Kinder weniger weitere Personen infizieren als erwachsene Indexfälle.

\section{Zahlen, Daten, Fakten}

Bisher sind in Österreich ca. 35.000 Personen positiv auf SARS-CoV-2 getestet worden. Davon waren $500(1,4 \%)$ unter 5 Jahre alt und 1600 (4,6\%) zwischen 5 und 14 Jahre alt. Während zu Beginn der Schulferien (Anfang Juli) 3,7\% der bis dahin positiv getesteten Personen Kinder (unter 14 Jahren) waren, ist dieser Anteil bis Schulbeginn im September auf $6 \%$ der Gesamtinfizierten angestiegen. Oder - anders ausgedrückt - ist die Zahl der Gesamtinfizierten in den Sommermonaten um ca. $60 \%$ gestiegen, während die Zahl der Infizierten Kinder im selben Zeitraum um $165 \%$ angestiegen ist. Ob dieser überproportionale Anstieg der (nachgewiesenen) kindlichen Infektionen während der Ferienmonate durch unspezifische Schwankungen oder veränderte Teststrategien in unterschiedlichen Altersgruppen verursacht wurde oder einer tatsächlich veränderten Altersverteilung entspricht, lässt sich nicht sicher beantworten. Die Ferien-bedingte Schließung der Schulen hat aber offensichtlich NICHT zur Reduktion von kindlichen Infektionen geführt.

\section{Kontakt mit Kinderabteilungen}

Bemerkenswert ist, dass nur ein sehr geringer Teil (ca. $6 \%$ ) der SARS-CoV-2 infizierten Kinder Kontakt mit Kinder- 


\section{ÖGKJ Elternbrief}

\section{Verhinderung einer Virus-Ausbreitung}

Liebe Eltern!

"Corona" hat uns allen Probleme beschert, die wir bisher in dieser Form nicht gekannt haben.

Dies betrifft gleichermaßen alle Bevölkerungsgruppen sowie Gesundheits- und Bildungseinrichtungen, v. a. aber auch Eltern und Familien.

Die bevorstehende Herbst-Wintersaison kann nun zusätzliche Probleme dadurch bringen, dass

1) In dieser Zeit zahlreiche verschiedene Viruserkrankungen auftreten können

2) In dieser Zeit sich üblicherweise auch Influenza (sog. „echte Grippe“) ausbreitet

3) Das Infektionsrisiko für "Corona“ weiter besteht

Bedauerlicherweise lässt sich bei vielen „Infekten“ durch eine ärztliche Untersuchung nicht eindeutig festlegen, welcher Erreger die jeweiligen Krankheitssymptome verursacht.

Sogenannte „Erkältungskrankheiten“ sind in den allermeisten Fällen durch Viren bedingt. Temperaturwechsel und Körpernähe (Menschenansammlungen) können die Verbreitung derartiger Erkrankungen entscheidend begünstigen. Dies gilt für relativ harmlose „Schnupfenviren“ ebenso wie für Influenza und letztlich SARSCoronavirus-2.

Für die Herbst-Wintersaison 2020/21 wird es daher entscheidend sein, die Infektionsrate GENERELL niedrig zu halten.

Dies kann in erster Linie dadurch gelingen, dass ALLE Personen (und insbesondere auch Kinder) mit „Infekten“ Kontakte zu anderen Menschen möglichst vermeiden. Konkret bedeutet dies, dass in der kommenden Herbst-Wintersaison Kinder mit Infektzeichen GRUNDSÄTZLICH Kindergärten und Schulen NICHT besuchen sollen. Wenn diese Empfehlung KONSEQUENT eingehalten wird, kann auch insgesamt mit einer niedrigen Infektionsrate gerechnet werden. Kinder werden dann voraussichtlich nicht 5-10 „Infekte" pro Saison durchmachen, sondern nur 1-2, vielleicht auch gar keinen!

Eltern müssen dann auch deutlich weniger Pflegeurlaub wegen Erkrankungen ihrer Kinder nehmen.

Als Kinder- und JugendärztInnen rufen wir dringend dazu auf, im Sinne der Allgemeinheit die Regel „Kranke Kinder bleiben zu Hause“ konsequent zu befolgen und dadurch auch das „Coronaproblem“ besser unter Kontrolle zu halten.

abteilungen hatten. Bis Mitte September waren 115 Kinder und Jugendliche mit SARS-CoV-2 in (ambulanter oder stationärer) Betreuung an österreichischen Abteilungen für Kinderund Jugendheilkunde, davon 39 stationär und 6 in Behandlung auf Intensivstationen. Zum Zeitpunkt der Erhebung (Mitte September) waren 2 Kinder wegen einer SARS-CoV-2 Infektion stationär, eines davon, ein Neugeborenes, auf der Intensiv-Station. Seit Beginn der Pandemie wurden uns 7 pädiatrische PatientInnen mit einer überschießenden Immunreaktion gemeldet, dabei konnte bei 4 eine akute oder abgelaufene Infektion mit SARS-CoV-2 nachgewiesen werden, bei den übrigen 3 gelang ein solcher Nachweis nicht. In Österreich ist bisher kein Kind an oder mit einer Coronavirusinfektion verstorben.

\section{Schulstart: kein Grund zur Verunsicherung}

Der herbstliche Schulstart sorgt bei vielen für Verunsicherungen, viele befürchten eine vermehrte Verbreitung in den Schulen. Dabei geben sowohl die Analysen der österreichischen Cluster durch die AGES als auch internationale Studien u. a. aus Skandinavien, Großbritannien und Australien Grund zur Entwarnung: In Schweden sind trotz geöffneter Schulen gleich wenige Kinder infiziert wie in Finnland, wo die Schulen geschlossen wurden. In Großbritannien gab es im ersten Monat nach der Schulöffnung bei über einer Million Schülern nur 70 Corona-
Fälle. In Australien gab es in der Zeit vor den Ferien nur vereinzelte Fälle in Schulen und nur einen einzigen Cluster in einem Kindergarten - der Indexfall war dabei ein Erwachsener. Auch sonst zeigt sich, dass die Infektionsketten meist von Erwachsenen ausgehen und Kinder die Infektion seltener weitergeben.

„Während SARS-CoV-2 Infektionen also offensichtlich in Schulen und Kinderbetreuungseinrichtungen nicht überproportional auftreten, wird die Abgrenzung zwischen COVID-19 und anderen respiratorischen Infektionen bei Kindern im Herbst eine große Herausforderung", beendete Strenger seine Ausführungen.

\section{Impfen ist jetzt wichtiger denn je} $\mathrm{Zu}$ den bedeutendsten Kollateralschäden der "Coronakrise" zählt ein weiteres Absinken der schon zuvor durchwegs zu niedrigen Durchimpfungsraten, bedingt durch eine zwar rational unbegründete, aber dennoch nachhaltige Angst vor Ansteckung beim Arztbesuch.

„In Österreich", berichtete Univ.Doz. Dr. Hans Jürgen Dornbusch, Referatsleiter der Impfkommission der ÖGKJ, „lagen die Impfraten während des „Lockdown“ im letzten Frühjahr regional unterschiedlich um $25 \%$ bis $90 \%$ unter jenen des Vergleichszeitraumes im Vorjahr. Die WHO kalkuliert in diesem Zusammenhang, dass im Zuge der SARS-CoV-2-Pandemie weltweit zusätzlich über 100 Millionen Kinder keine oder nur eine Impfung gegen Masern erhalten haben."

Durch die große Anzahl an Ungeimpften drohen nicht nur neuerliche Masernausbrüche, eine Zunahme von potentiell lebensbedrohlichen Erkrankungen wie Keuchhusten oder schweren Pneumokokken- und Meningokokkeninfektionen und zeitversetzt zahlreiche verhinderbare HPV-assoziierte, vielfach bösartige Tumore, sondern auch das Wiederauftreten verschwunden geglaubter Infektionskrankheiten wie z. B. Diphtherie. Ärzte- 
Hier steht eine Anzeige.

Springer 
schaft und Gesundheitsbehörden sind daher aufgerufen, die entstandenen Impflücken möglichst rasch durch vermehrte Aktivität und zusätzliche Informationskampagnen zu schließen, und dies wird bereits in konzertierter Weise umgesetzt.

„Das für die kommende HerbstWinter-Saison besonders wichtige Thema "Influenza \& Corona" wird", so Dornbusch, „in Kürze anlässlich einer eigenen Pressekonferenz mit detaillierten Informationen zum geplanten kostenfreien Influenza-Impfprogramm behandelt werden."

\section{Die Eltern haben es in der Hand} Die wirkungsvollste Methode zur Verhinderung einer Virusverbreitung ist das sogenannte "Containment". Darunter versteht man, dass infizierte Personen möglichst separiert/isoliert werden, um so eine Weiterverbreitung zu verhindern.„Dies gilt gleichermaßen für "Schnupfenviren", Influenza und SARS-CoV-2", ergänzte Prim. Univ. Prof. Dr. Reinhold Kerbl, Vorstand der Abteilung für Kinder und Jugendliche am LKH Hochsteiermark/Leoben, Generalsekretär der ÖGKJ. Die ÖGKJ appelliert daher an Eltern und Familien, ihren Teil zur Viruseingrenzung (damit sind alle „Infektviren“ gemeint) beizutragen. -
Dazu hat die ÖGKJ einen Elternbrief herausgegeben (s.u.).

\section{Weitere Informationen:}

www.paediatrie.at

Hinweis des Verlags. Der Verlag bleibt in Hinblick auf geografische Zuordnungen und Gebietsbezeichnungen in veröffentlichten Karten und Institutsadressen neutral.

Paediatr. Paedolog. 2020 · 55:269-272 https://doi.org/10.1007/s00608020-00838-6

(c) Springer-Verlag GmbH Austria, ein Teil von Springer Nature 2020

Hier steht eine Anzeige. 